Goodin, D. S., Souires, K. C. Henderson, B. H., Starr, A. (1978) Age-related variations in evoked potentials to auditory stimuli in normal human subjects. Electroencephalography and Clinical Neurophysiology, 44, 447-458.

Pferferbaum, A., Wenwgrat, B. G., Ford, J. M., Roth, W. T. \& KOPELL, B. S. (1984) Clinical application of the P3 component of event-related potentials. II. Dementia, depression and schizophrenia. Electroencephalography and Clinical Neurophysiology, 59, 104-124.

Romani, A., Mariott, G. \& Cosı, V. (1986) The effect of aging on the $\mathrm{P} 3$ component in different auditory paradigms. Revue d'Electroencephalographie et de Neurophysiologie Clinique, 16, 423-431.

—. TAva, G., APIH, G., Cosi, V. (1987) Some relationships between spectral EEG parameters and late AEPs. Bollettino della Societa Italiana di Biologia Sperimentale (in press).

WeSterkAMP, J. J. \& AUNON, J. I. (1987) Optimum multielectrode a posteriori estimates of single-response evoked potentials. IEEE Transactions on Biomedical Engineering, 34, 13-22.

\section{Alcohol and Ageing}

SIR: Nordstrom \& Berglund (Journal, September, $1987,151,382-388$ ) fail to provide vitally important information about what would otherwise be one of the few tangible pieces of information resulting from their follow-up study of male alcoholics. They state that they wish to examine the issue of ageing and recovery from alcoholism. However, in their final analysis of $\mathbf{4 5}$ males selected from an original mixedsex sample of 1312 patients, they state, "The main finding of the present study was that the processes of improvement differed between older and younger alcoholics. Improvement in older subjects was related to a pattern of gradual change from abuse to social drinking." The authors examine three possible physiological mechanisms to explain their observations: two of these they were only able to speculate about in their patients, and the third - the possibility of liver damage causing decreased alcohol tolerance and a gradual reduction in alcohol consumptionthey dismiss with the bland statement that "while subjects with pathological liver function blood tests in our sample were typical abusers, there was no indication of liver damage in the improved subjects". What evidence do the authors have that their improved subjects did not have liver damage? It is well-recognised that alcoholic liver damage may be present in the reformed non-abusing patient even if the routine laboratory liver function tests are normal. The only way to be sure whether or not the patient has suffered permanent liver damage is by histopathological examination of a liver biopsy (Sherlock, 1985).

Hill End Hospital

M. A. ROBERTS

St Albans

Herts ALA ORB

\section{Reference}

SHERLOCK S. (1985) Alcohol and the liver. In Diseases of the Liver and Biliary System (ed. Dame S. Sherlock), p 355. London: Blackwell Scientific Publications.

SIR: As is correctly pointed out by Dr Roberts, we cannot exclude the possibility of liver damage in the absence of pathological test results in our subjects. Apart from liver function blood tests at the followup, however, we also studied all psychiatric and several somatic hospital case records concerning our subjects. We were interested, among other things, in data concerning somatic complications. In short, we found no evidence of liver damage in the group of subjects referred to in our paper as improved, whereas some of the subjects with an unsuccessful course had been under medical treatment for liver damage. Although this does not prove anything about absence or presence of liver damage in any of our subjects, we still feel that, on a group level, our suggestion that "severe liver disease. .. does not seem to be a plausible explanation for improvement in the present sample" is reasonable and justified.

GÖRAN NORDSTRÖM MATS BERGLUND

University of Lund

$S$-221 85 Lund

Sweden

\section{Constant Current vs Constant Voltage ECT Devices}

SIR: Railton et al (Journal, August 1987, 151, 244-247) report a comparison of various electrical parameters measured during clinical use with Ectron Duopulse Mark 4 and Ectron Series 2A ECT devices. They then go on to interpret their findings on the basis of differences in the mode of stimulus delivery between these two machines, i.e. constant voltage for the former and constant current for the latter. In fact, the mode of stimulus delivery is only one of two major differences between these two machines, the other being stimulus waveform: partial sine wave for the Duopulse and brief pulse for the Series 2A devices.

Various investigations have demonstrated that the sine wave stimulus requires several times more stimulus energy and charge to produce a seizure than does the pulse stimulus (Weiner, 1980). By extrapolation, it is reasonable to assume that the pulse stimulus is also more efficient in inducing seizures than the $60 \%$ sine wave stimulus used in the Ectron Duopulse. This difference in seizure threshold, which appears to be independent of mode of stimulus delivery, therefore means that in order to assure the occurrance of 
adequate seizures, a higher energy output is required for the Duopulse device. Decreasing the intensity or duration of the partial sine wave stimulus, as suggested by the authors, would be likely to have the effect of diminishing its ability to generate an adequate ictal response.

This possibility is an important consideration, since the output of both devices may be marginal with respect to patients with high seizure thresholds, e.g. the elderly. Experience with contemporary American pulse ECT devices has shown, in this regard, that brief pulse seizure thresholds between 50 and $100 \mathrm{~J}$ are not rare. On the other hand, such experience has also made it clear that seizure thresholds in other patients may be as low as $5 \mathrm{~J}$, suggesting that fixed parameter devices such as the Ectron Duopulse may not be as optimal in this regard as newer generation equipment by Ectron, Elcot, Mecta, Medcraft, and Somatics (the latter four of US origin).

With respect to the issue of constant voltage versus constant current stimulation, it can be said that the latter appears to be a more physiological means of stimulation in terms of neuronal depolarisation. In addition, as we have pointed out elsewhere (Weiner \& Coffey, 1986), relative differences between constant current and constant voltage stimulation also exist concerning the effects of inter-electrode resistance. A high resistance, particularly likely in the elderly, may result in insufficient current delivery with a constant voltage device. A low resistance, however, possibly on the basis of increased shunting of current across the scalp, will be associated with a relatively high current with the constant voltage apparatus. While this latter effect may allow the constant voltage device to compensate for increased scalp shunting, it also raises the likelihood of skin burns in such cases.

RichaRd D. WeINER

Duke University Medical Center

Durham, North Carolina 27710

USA

\section{References}

WEINER, R. D. (1980) ECT and seizure threshold: effects of stimulus wave form and electrode placement. Biological Psychiatry, 15, 225-241.

- \& Cofrey, C. E. (1986) Differential ability of ECT devices to produce seizures. Convulsive Therapy, 2, 134-135.

SIR: Dr Weiner has published data showing lower seizure thresholds for patients receiving a brief stimulus compared with patients receiving sine-wave stimulus (Weiner, 1980). This supports the assertion that more energy may be required for a sine-wave stimulus than for a pulse stimulus. However, in $\mathrm{Dr}$ Weiner's investigation, he used equipment which produces a continuous sine wave for short duration (Medcraft B-24 MK III). The important difference between this machine and the Ectron Duopulse MK IV used in our study is that in the latter machine, the sine wave is truncated by a thyristor switch. This means that there is a rapid change in voltage similar to the rising edge of the pulses in the Ectron constant current machine. These waveforms can be seen in Fig. 2 of our paper. It is therefore not valid to assume that results obtained with a pure sine-wave stimulus are relevant to a truncated sine-wave stimulus.

\section{R. RAILTON}

\section{Monklands District General Hospital \\ Monkscourt Avenue}

Airdrie ML6 0JS

\section{Reference}

WEINER, R. D. (1980) ECT and seizure threshold: effects of stimulus wave form and electrode placement. Biological Psychiatry, 15, 225-241.

\section{Clonidine and Neuroleptic-Resistant Mania}

SIR: Maguire \& Singh (Journal, June 1987, 150, 863-864) report the efficacy of clonidine in three cases of haloperidol-resistant mania. In the light of our experience, we would like to stress two points: firstly, the anti-manic effect of clonidine is already documented - our team at the Salpetrière reported the anti-manic effect of this compound as early as 1980 (Jouvent et al, 1980) and, more recently, we published a study bearing on 24 patients; secondly, the latter study showed that a poor previous response to neuroleptic drugs seemed to be a predictor of clonidine efficacy (Hardy et al, 1986).

These facts, and the three supplementary cases published by Maguire \& Singh, are not necessarily in favour of the 'noradrenergic hypothesis of mania', but may indicate that some patients who do not respond to neuroleptic medication may have an NA hyperactivity which benefits from NA reduction by clonidine.

R. JOUVENT
Y. LECRUBIER
M-C. HARDY
D. WIDLOCHER

Hopital de La Salpétrière 47 Bd de l'Hopital 75013, Paris 